\title{
Impact of Treatment-Related Beliefs on Medication Adherence in Immune-Mediated Inflammatory Diseases: Results of the Global ALIGN Study
}

\author{
Pierre Michetti · John Weinman · Ulrich Mrowietz · Josef Smolen • \\ Laurent Peyrin-Biroulet - Edouard Louis - Dieter Schremmer • \\ Namita Tundia · Pascal Nurwakagari · Nicole Selenko-Gebauer
}

Received: September 1, 2016 / Published online: November 16, 2016

(C) The Author(s) 2016. This article is published with open access at Springerlink.com

\begin{abstract}
Introduction: Medication adherence is critical in chronic immune-mediated inflammatory diseases (IMIDs) and could be affected by patients' treatment-related beliefs. The objective of this study was to determine beliefs about systemic medications in patients with
\end{abstract}

Enhanced content To view enhanced content for this article go to http://www.medengine.com/Redeem/ 2617F0604A1D4360.

Electronic supplementary material The online version of this article (doi:10.1007/s12325-016-0441-3) contains supplementary material, which is available to authorized users.

P. Michetti $(\bowtie)$

Crohn and Colitis Centre, Gastro-entérologie La

Source-Beaulieu and Division of Gastroenterology,

Centre Hospitalier Universitaire Vaudois, 1004

Lausanne, Switzerland

e-mail: pmichetti@gesb.ch

J. Weinman

Institute of Pharmaceutical Sciences, King's College

London, London, UK

U. Mrowietz

Psoriasis-Center at the Department of Dermatology,

Venereology and Allergology, University Medical

Center Schleswig-Holstein, Campus Kiel, Kiel,

Germany
IMIDs and to explore the association of those beliefs and other factors with adherence.

Methods: This was a multi-country, cross-sectional, self-administered survey study. Included were adults diagnosed with one of six IMIDs receiving conventional systemic medications and/or tumor necrosis factor inhibitors (TNFi). Patients' necessity beliefs/concerns towards and adherence to treatments were assessed by the Beliefs about Medicines Questionnaire and four-item Morisky Medication Adherence Scale. Correlation of patients' beliefs about treatment and other factors with adherence were evaluated by multivariable regression analyses.

\footnotetext{
J. Smolen

Division of Rheumatology, Department of Medicine

3 , Medical University of Vienna, Vienna, Austria

J. Smolen

2nd Department of Medicine, Center for Rheumatic Diseases, Hietzing Hospital, Vienna, Austria

L. Peyrin-Biroulet

Inserm U954 and Department of Gastroenterology, Université de Lorraine, Vandoeuvre-les-Nancy,

France

E. Louis

CHU de Liège et Université de Liège, Liège, Belgium
} 
Results: Among studied patients $(N=7197)$, $32.0 \%$ received TNFi monotherapy, $27.7 \%$ received TNFi-conventional combination therapy, and $40.3 \%$ received conventional medications. Across IMIDs, high adherence to systemic treatment was more prevalent in TNFi groups (61.3-80.7\%) versus corresponding conventional treatment groups (28.4-64.7\%). In at least four IMIDs, greater perception of the illness continuing forever $(P<0.001)$, of the treatment helping $(P<0.001)$, and more concerns about the illness $(P<0.01)$, but not clinical parameters, were associated with higher treatment necessity beliefs. Higher treatment necessity beliefs, older age, Caucasian race, and TNFi therapy were associated with high medication adherence in at least four IMIDs.

Conclusions: Treatment necessity beliefs were higher than concerns about current medication in patients with IMID. Illness perceptions had a greater impact on treatment necessity beliefs than clinical parameters. Older age, greater treatment necessity beliefs, and TNFi therapy were associated with high self-reported medication adherence in at least four IMIDs.

Trial registration: ACTRN12612000977875.

Funding: AbbVie.

Keywords: Crohn's disease; Cross-sectional study; Illness perception; Medication beliefs; Rheumatoid arthritis; Ulcerative colitis

D. Schremmer

GKM Gesellschaft für Therapieforschung $\mathrm{mbH}$,

Munich, Germany

N. Tundia

AbbVie Inc., North Chicago, IL, USA

P. Nurwakagari

Medical Department, AbbVie Deutschland GmbH \&

Co. KG, Wiesbaden, Germany

N. Selenko-Gebauer

Global Medical Affairs, AbbVie Inc., North Chicago, IL, USA

\section{INTRODUCTION}

Treatment adherence is of particular importance in immune-mediated inflammatory diseases (IMIDs), as these chronic conditions require long-term management. Non-adherence to treatment in patients with IMIDs has been shown to negatively impact patient outcomes, such as increased disease activity or reduced treatment efficacy [1-3], increased risk of clinical recurrence ("flares") [4-6], lower quality of life [7], and risk of hospitalization or prolongation of hospital stay [8-10], resulting in increased healthcare resource use and costs [8-12].

According to the World Health Organization, adherence to long-term therapy is defined as the degree to which a person's use of medication, following a diet, and/or adopting lifestyle changes is consistent with agreed-upon recommendations from a healthcare provider [13]. Many patients do not, however, take their medication as prescribed, or their adherence to therapy fluctuates over time, engendering difficulties in monitoring patient adherence by the treating physician. Depending on the methodology of adherence measurement and received medication, studies reveal non-adherence to prescribed therapies in a large proportion of patients with IMIDs (reported adherence rates: psoriasis [PS], 22-67\% [14]; rheumatoid arthritis [RA], 21-99\% [1, 2, 15-21]; Crohn's disease [CD] or ulcerative colitis [UC], 28-96\% $[4,10,15,22,23])$. Currently, it is unclear whether lack of adherence relates to particular diseases and therapies or is, in principle, similar across diseases and types of treatment. Different diseases affect patients to a greater or lesser extent, and differences in age, disease severity and duration, and beliefs about medications also may have an effect on adherence to 
therapy. The ALIGN study was designed to address these questions.

Non-adherence to therapy appears to be intentional in the majority of cases, as only $12 \%$ of patients with chronic diseases report problems remembering to take their medication (unintentional non-adherence) [24]. Studies examining long-term conditions have shown that intentional non-adherence may result in part from doubts about the real need for and expected benefit from a certain medication, paired with concerns about its potential side effects and ability to improve long-term outcomes [25-27]. These overall attitudes towards medications are subjective, relate to patients' overall trust in their medicines, and have been shown to differ, even in groups of patients treated with the same medication for the same condition [28].

The primary objective of the ALIGN study was to assess necessity beliefs and risk concerns in patients with any of six chronic IMIDs towards their systemic medication. Secondary objectives were to assess adherence to different IMID treatments, to determine the factors affecting patients' beliefs about systemic medications, and to explore the association between patients' beliefs about treatments and other factors with medication adherence in patients with IMIDs.

\section{METHODS}

ALIGN was a global, multi-country, cross-sectional, self-administered survey study conducted between June 2012 and October 2013 that enrolled 7328 patients from 501 sites in six geographic areas (Fig. S1); data from 7197 patients were used in this analysis.

\section{Patients}

Patients included in ALIGN were at least 18 years old and attended routine outpatient visits. All patients had either RA, ankylosing spondylitis (AS), psoriatic arthritis (PsA), PS, CD, or UC diagnosed by a rheumatologist (RA, PsA, and AS), dermatologist (PsA and PS), or gastroenterologist (CD and UC), respectively, and were being treated with systemic conventional disease-modifying antirheumatic drugs (DMARDs), immunosuppressants, glucocorticoids, nonsteroidal anti-inflammatory drugs (NSAIDs; for patients with AS only), and/or TNFi. Current and prior disease severity was rated by the investigator on a five-point scale (mild, mild to moderate, moderate, moderate to severe, or severe). Treatment response was evaluated by the investigator (complete response, partial response, and not evaluable).

All procedures followed were in accordance with the ethical standards of the responsible committee on human experimentation (institutional and national) and with the Helsinki Declaration of 1964, as revised in 2013. Informed consent for participation was obtained from all patients included in the study. This study was approved by the local ethics committee of each participating country.

\section{Study Procedures}

Consecutive patient recruitment was carried out if patients agreed to participate in the study. Data collection occurred at a single visit during routine follow-up. Patients were asked to complete four validated questionnaires: the Beliefs about Medicines Questionnaire (BMQ) [29], scoring treatment necessity beliefs and concerns on a 5 -point Likert scale $(1=$ strongly disagree; $5=$ strongly agree; Table S1a, b); the four-item Morisky Medication Adherence Scale 
(MMAS-4) [30, 31], consisting of four questions (yes $=0 ;$ no $=1$ ), with high adherence defined as a score of 4 ; the Brief Illness Perception Questionnaire (BIPQ; Table S1c) [32], measuring perception of illness with eight questions scored using an 11-point scale [0-10]); and Patient Health Questionnaire-2 (PHQ-2) [33], which consists of two questions measuring depressive symptoms, scored on a 4-point rating scale ( $0=$ not at all; $3=$ nearly every day). Validated translations were provided in the languages of patients expected to enroll. The questionnaires were completed on paper and placed in sealed envelopes by the patient to guarantee confidentiality. The investigators collected information on patient demographics, social, economic, and educational background, IMID-related data, prior and current treatment, and response to therapy. Comorbidity was collected from the patient files or by interviewing the patient and recorded in the case report form by the investigator during the documentation visit. Comorbidity was then coded using the Medical Dictionary for Regulatory Activities, version 16.1.

\section{Statistical Analyses}

Data analysis was conducted using information from 7197 of 7328 enrolled patients who had available data for at least one of the patient questionnaires used in the study. Current treatments were categorized as TNFi monotherapy ("TNFi mono"), TNFi combined with conventional therapies ("TNFi combo"), or conventional therapies only ("conventional only"). DMARDs, immunosuppressants, glucocorticoids, and NSAIDs (only allowed in patients with AS) were classified as conventional therapy. BMQ-Specific Necessity and Concerns subscales ratings and the proportion of highly adherent patients (defined as MMAS- $4=4$ ) were estimated in the TNFi monotherapy group, the TNFi combo groups, and the conventional only group. This definition of high adherence was the same as for the original Morisky, Green, and Levine adherence scale, in which a score of 4 had good predictive validity for adherence in patients with high blood pressure [34]. BMQ ratings and MMAS-4 adherence rates in the TNFi combo group were evaluated separately for the TNFi component ("TNFi combo-TNFi rating") and the conventional therapy component ("TNFi combo-conventional rating"), as beliefs and adherence patterns might differ. No statistical comparisons of differences between treatment groups were performed.

Multivariable regression analyses were employed to evaluate the factors affecting BMQ-Specific Necessity/Concerns scores and medication adherence. Covariates in these analyses consisted of age, gender, disease duration, current disease severity, prior disease severity, treatment response (complete vs non-complete), treatment duration, number of pretreatments, BIPQ1-BIPQ8 subscores, PHQ-2 total score, number of concomitant diseases, education (years), living arrangement (living alone, living with partner, or living with others), residence (urban vs rural), and Caucasian ethnicity (Caucasian vs non-Caucasian). All demographic and clinical data were collected from the patient files or by interviewing the patients. Regression analyses were performed separately for all six indications. A backward selection approach, based on the predicted residual sum of square (PRESS) criteria and removal when $P>0.05$, was used in multivariable linear and logistic regression analyses to determine predictors of high BMQ-Specific scores and high medication adherence. To account for the within-subject 
correlations, the final model was refitted by a random effects linear model with a patient indicator as a random intercept. To prevent overfitting of the data, the model with the smallest prediction error (minimal PRESS estimate) was selected as the final model. All statistical analyses were performed using SAS 9.2 (SAS Institute, Cary, NC, USA).

\section{RESULTS}

\section{Disease and Patient Characteristics}

\section{Patient Demographics}

ALIGN enrolled 7328 patients from 34 countries; data from 7197 patients enrolled in 33 countries who provided documentation for at least one questionnaire were used in the analysis. Patients were recruited from four different regions (Fig. S1); $56.8 \%$ of the patients were recruited in Western Europe and Canada. The percentages of female patients in the study varied depending on the IMID; mean ages ranged from 38.0 years $(\mathrm{CD})$ to 54.8 years (RA; Table 1). Additional patient characteristics are summarized in Table $\mathbf{S 2}$.

\section{Disease Characteristics}

The mean duration of disease varied across the six IMIDs (Table 1). Prior to the start of current IMID treatment, $68.8 \%$ of the patients had a disease severity rated as moderate to severe or severe, whereas under their current therapies, only $12.2 \%$ had disease severity rated as moderate to severe or severe (Table 1). A statistically significant improvement in patients' disease severity, from before the start of current treatment to the current state, was reported by the investigators $(P<0.0001$; Wilcoxon test), corresponding to an improvement of disease severity in $80.3 \%$ of patients, no change in $15.4 \%$, and worsening in $4.3 \%$.

A large proportion of patients were suffering from comorbidity, with the highest proportion in the RA population (66.9\%; Table 1). Vascular, metabolic and nutritional, and musculoskeletal and connective tissue disorders were common in patients receiving therapies for IMIDs; hypertension was the most common comorbidity across all IMIDs.

\section{IMID Medications}

Current IMID treatments were generally evenly distributed between TNFi monotherapy, TNFi combination therapy, and conventional DMARDs in patients with PsA and CD, whereas TNFi monotherapy was predominant in patients with PS (56.9\%) and AS (45.0\%), and conventional systemic treatments (DMARDs, immunosuppressants, glucocorticoids, or [only for patients with AS] NSAIDs) were predominant in patients with RA (58.7\%) and UC (54.9\%; Table 1). Additional treatment details are in Table S2.

\section{Beliefs Towards Medication and Treatment Adherence}

\section{Specific Beliefs Towards Current IMID Medications}

BMQ-Specific Necessity subscale scores indicated a relatively high perceived need for current treatment (Fig. 1a; Table S3a). Numerically higher mean scores were reported for patients receiving TNFi (either as monotherapy or as a combination therapy) compared with conventional therapy alone. BMQ-Specific Concerns subscale scores were lower than those observed for the BMQ-Specific Necessity subscale, and were in a similar range across the three treatment groups (Fig. 1a, b; Tables S3a, b). 


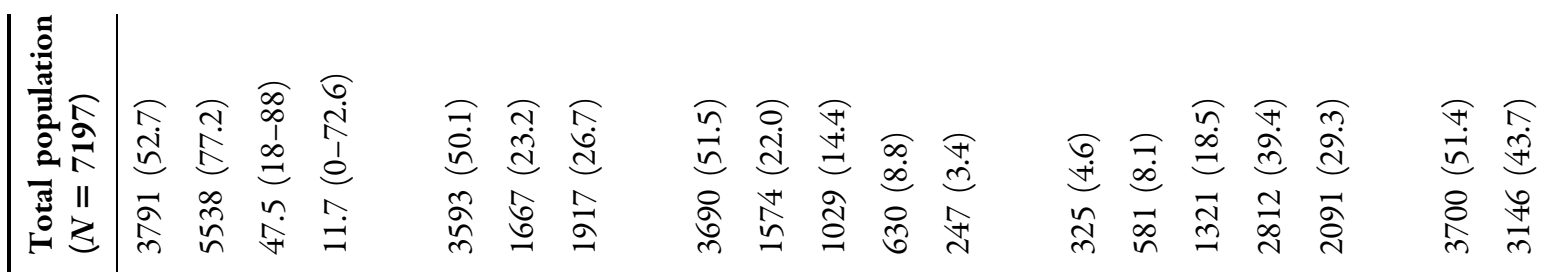

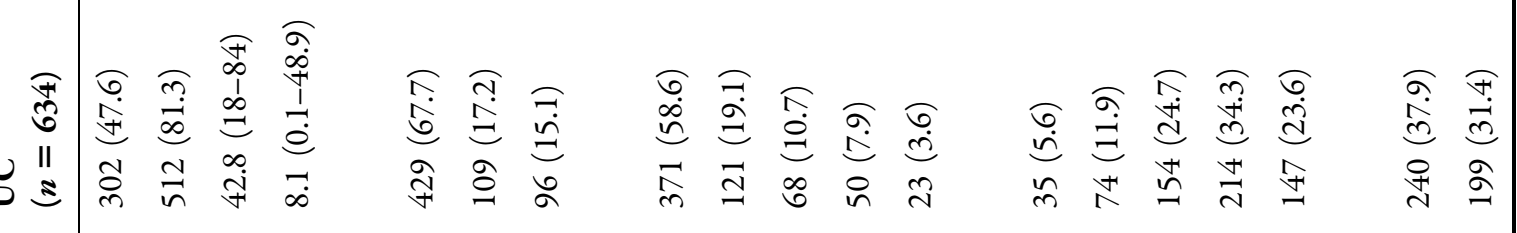

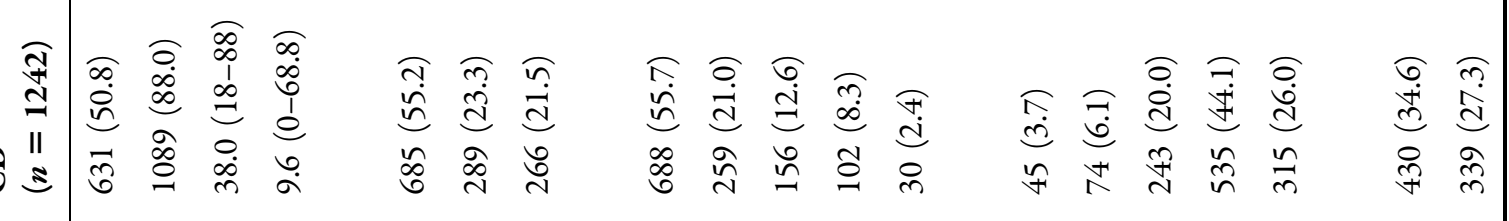

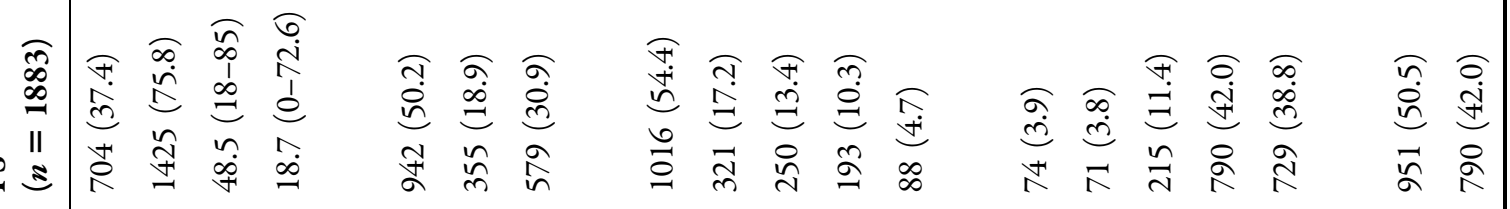

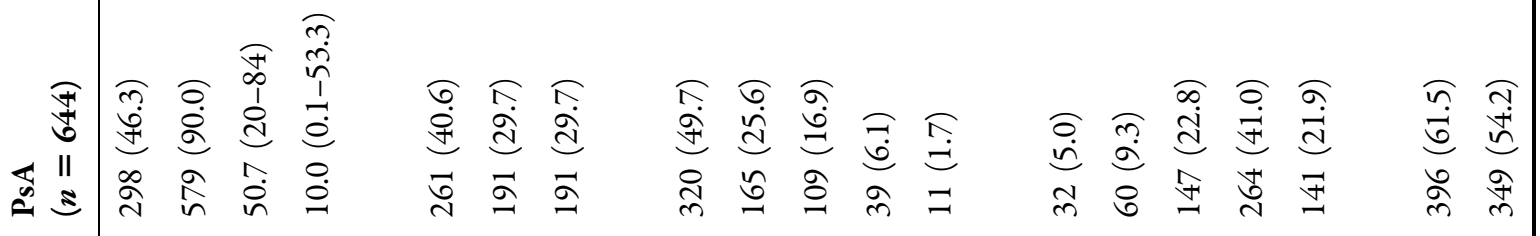

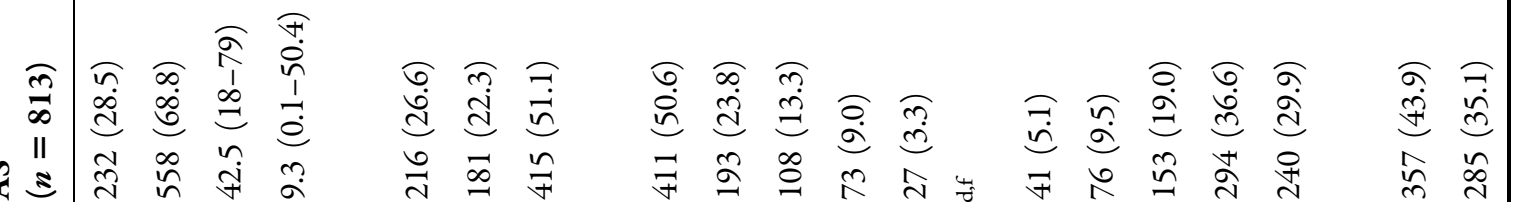

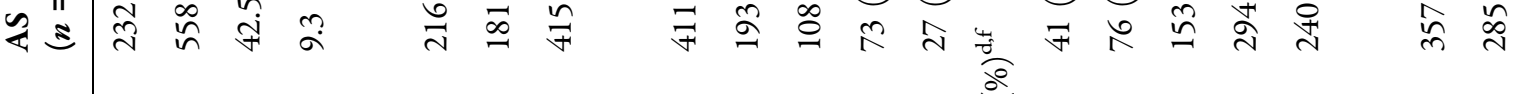

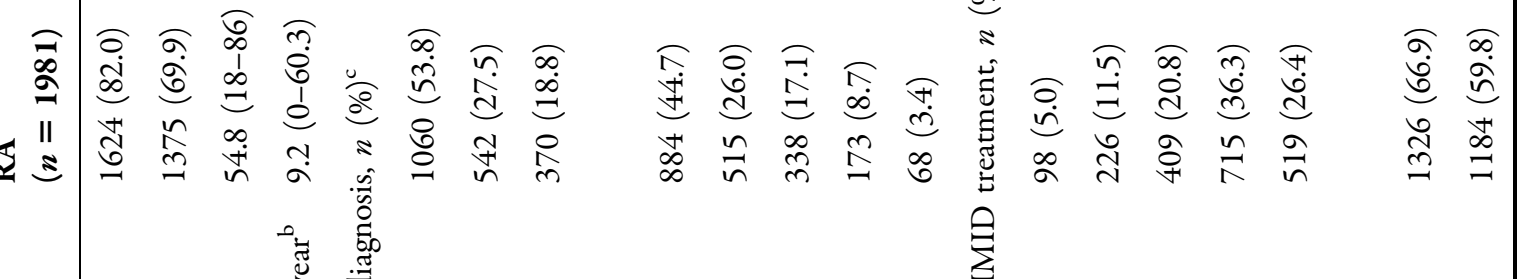

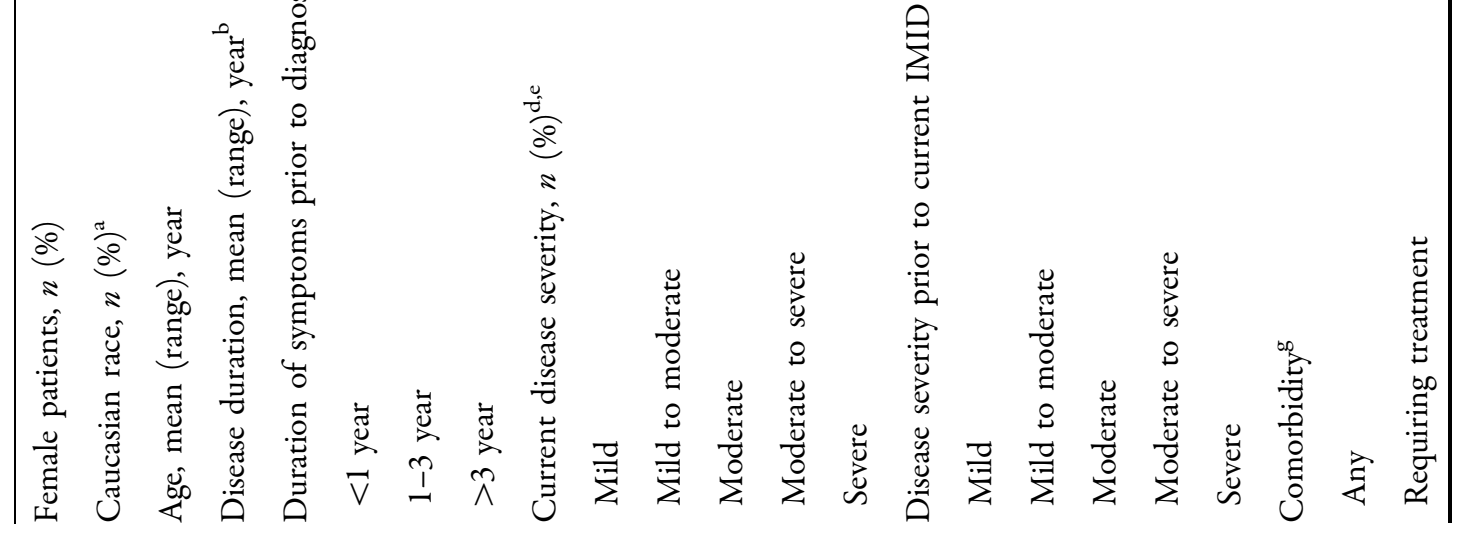




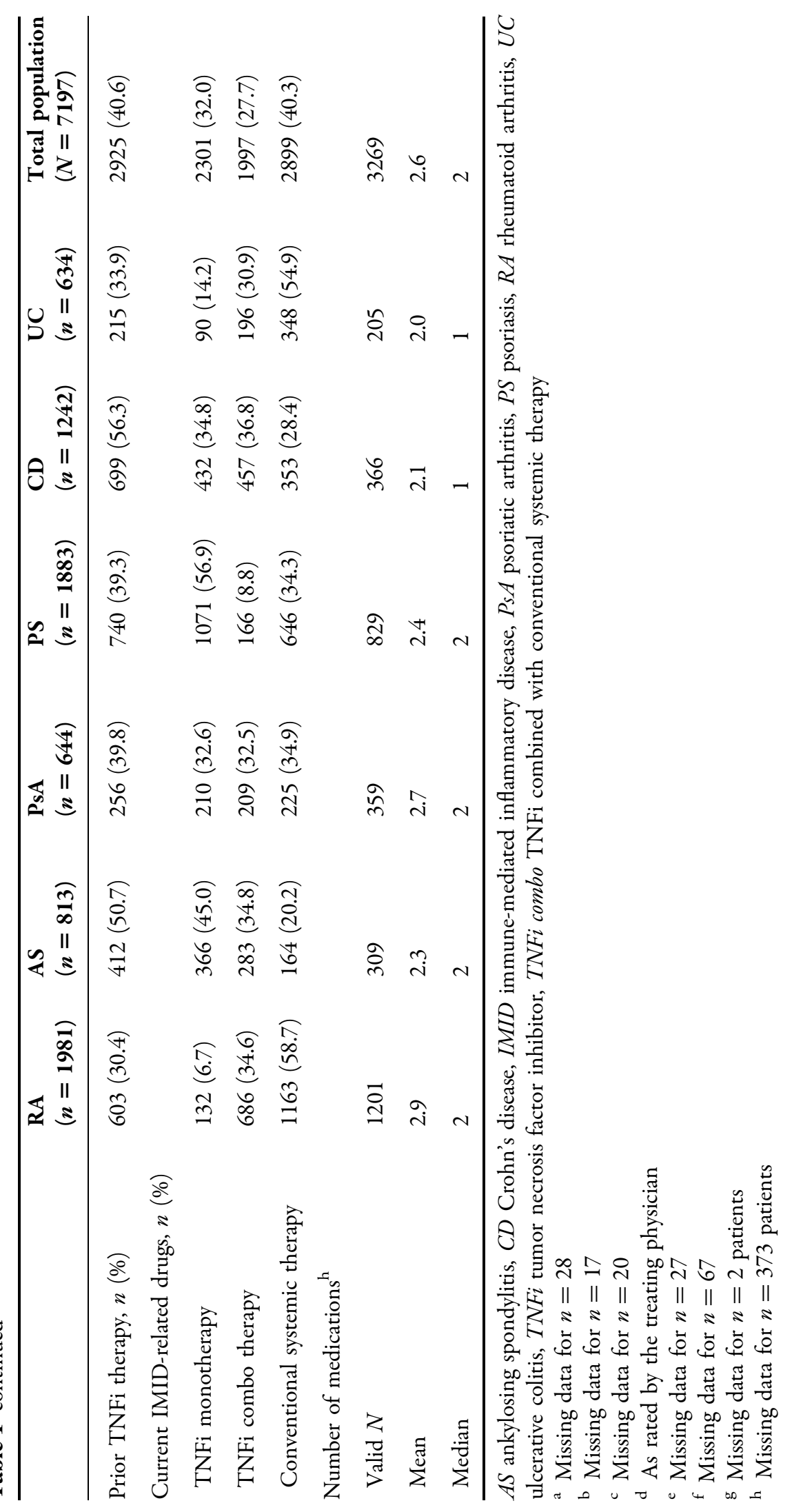



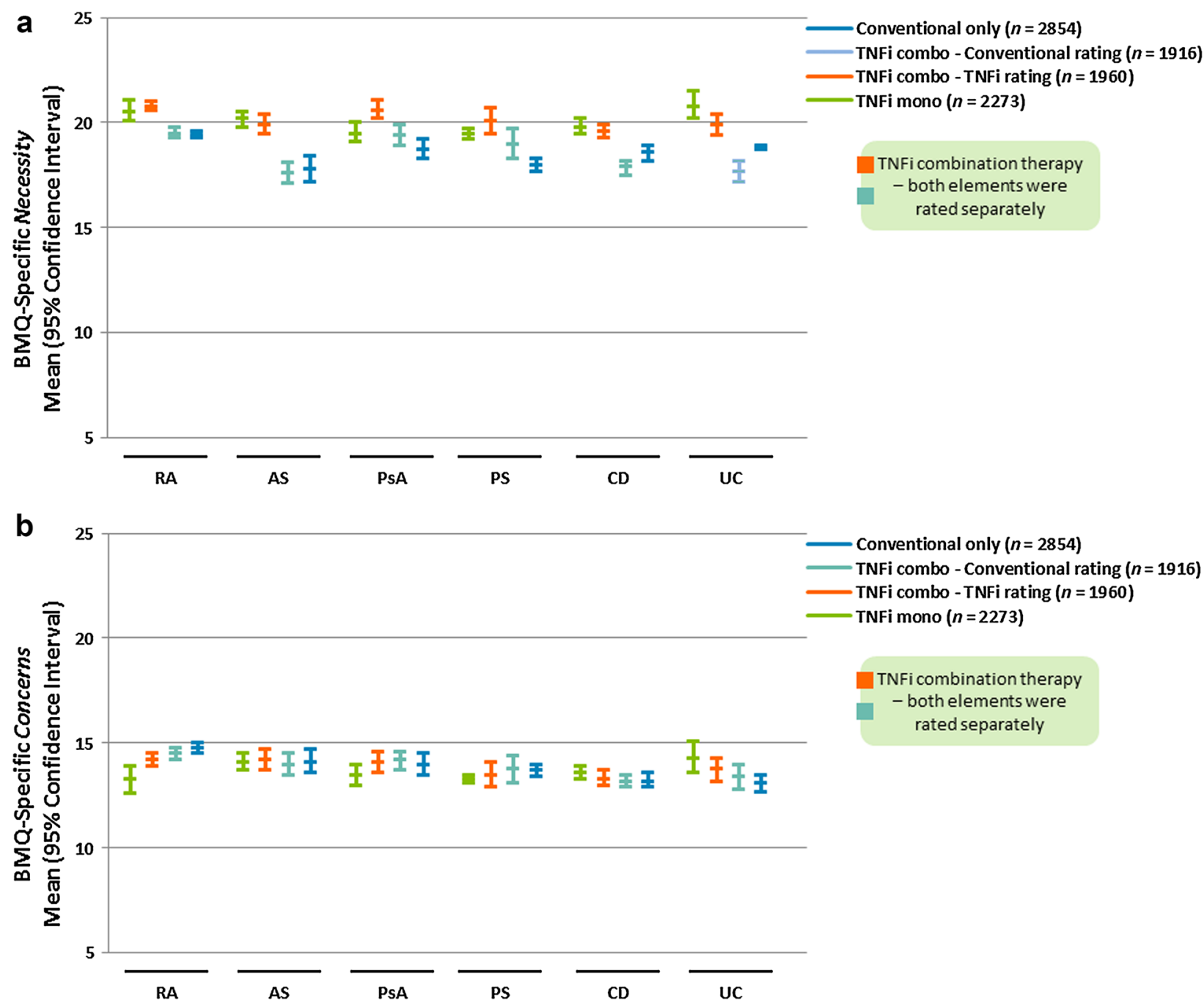

Fig. 1 Mean with lower and upper 95\% confidence interval values for BMQ-Specific subscales (a) Necessity and (b) Concerns by IMID diagnosis and treatment group. $A S$ ankylosing spondylitis, $B M Q$ Beliefs about Medicines Questionnaire, $C D$ Crohn's disease, IMID immune-mediated inflammatory disease, $P s A$ psoriatic

\section{Beliefs About Medications in General}

According to BMQ-General subscale results covering general medication overuse and harm beliefs (Table S1b), we found a significant proportion of patients with negative beliefs about medication in general (e.g., perception of "all medicines to be poison" [9.0-20.3\% of patients] and of "most medicines to be arthritis, $P S$ psoriasis, $R A$ rheumatoid arthritis, $T N F i$ tumor necrosis factor inhibitor, $U C$ ulcerative colitis. Missing data for $n=28$ (TNFi mono), $n=37 \quad$ (TNFi combo - TNFi rating), $\quad n=81$ (TNFi combo - Conventional rating), and $n=45$ (Conventional only)

addictive" [14.6-24.0\%]). These beliefs were highest in patients with RA and were lowest in patients with CD and UC (Table S4). Agreement with the belief that "physicians place too much trust on medicines" ranged from $28.4 \%$ to $45.6 \%$, depending on the specific IMID. Up to $28.2 \%$ of patients believed that they "should stop their treatment for a while every now and 


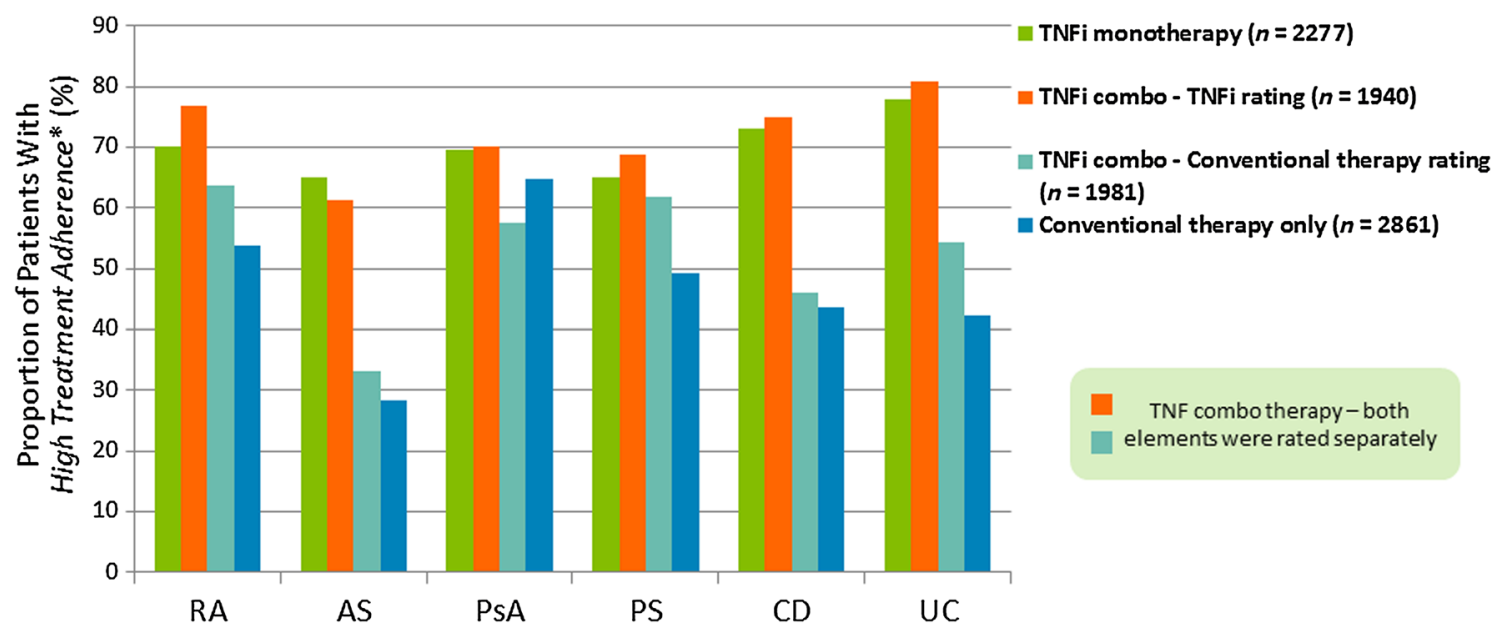

Fig. 2 Self-reported adherence in patients on TNFi monotherapy, TNFi combination therapy, or conventional therapy by MMAS-4. *Patients with high adherence were defined as those with an MMAS-4 score $=4$. AS ankylosing spondylitis, $B M Q$ Beliefs about Medicines Questionnaire, CD Crohn's disease, IMID immune-mediated inflammatory disease, $M M A S-4$ four-item Morisky Medication Adherence Scale,

then"; patients with PS agreed with this statement most frequently, and patients with $\mathrm{CD}$ and UC had the lowest agreement rate.

\section{Illness Perception and Depressive Symptoms}

Patients understood the chronic nature of their diseases, as reflected by high mean scores across IMIDs (8.5-9.0) in response to the BIPQ question "How long do you think your illness will continue?" Patients gave high mean BIPQ scores (8.3-8.5) for how much they thought that their treatment could help their illness. The lowest BIPQ scores were regarding how much their illness affected their life, how much they experienced symptoms, and how much their illness affected them emotionally (Table S5).

The percentage of patients with signs and symptoms suggestive of depression (PHQ-2 total
$P S$ psoriasis, $P s A$ psoriatic arthritis, $R A$ rheumatoid arthritis, $T N F i$ tumor necrosis factor inhibitor, $U C$ ulcerative colitis. TNFi mono valid data for $n=2277$, missing data for $n=24$, TNFi combo - TNFi rating valid data for $n=1940$, missing data for $n=57$, TNFi combo - Conventional rating valid data for $n=1918$, missing data for $n=79$, Conventional only valid data for $n=2861$, missing data for $n=38$

score $\geq 4)$ ranged from $13.7 \%(C D)$ to $20.5 \%$ (RA; Table S6).

\section{Self-Reported Adherence}

Across all indications, patients reporting high adherence to their current IMID treatment (MMAS-4 = 4) were more prevalent in TNFi-treated monotherapy and combination therapy groups (61.3-80.7\%) compared with conventional treatment groups (28.4-64.7\%; Fig. 2). For patients on TNFi combination therapy, the proportion of patients reporting to be highly adherent was greater for the TNFi component than for the conventional therapy component (Fig. 2). The lowest rate of highly adherent patients was observed in the AS population receiving conventional medication, either alone $(28.4 \%)$ or in combination therapy (33.1\%; Fig. 2). 


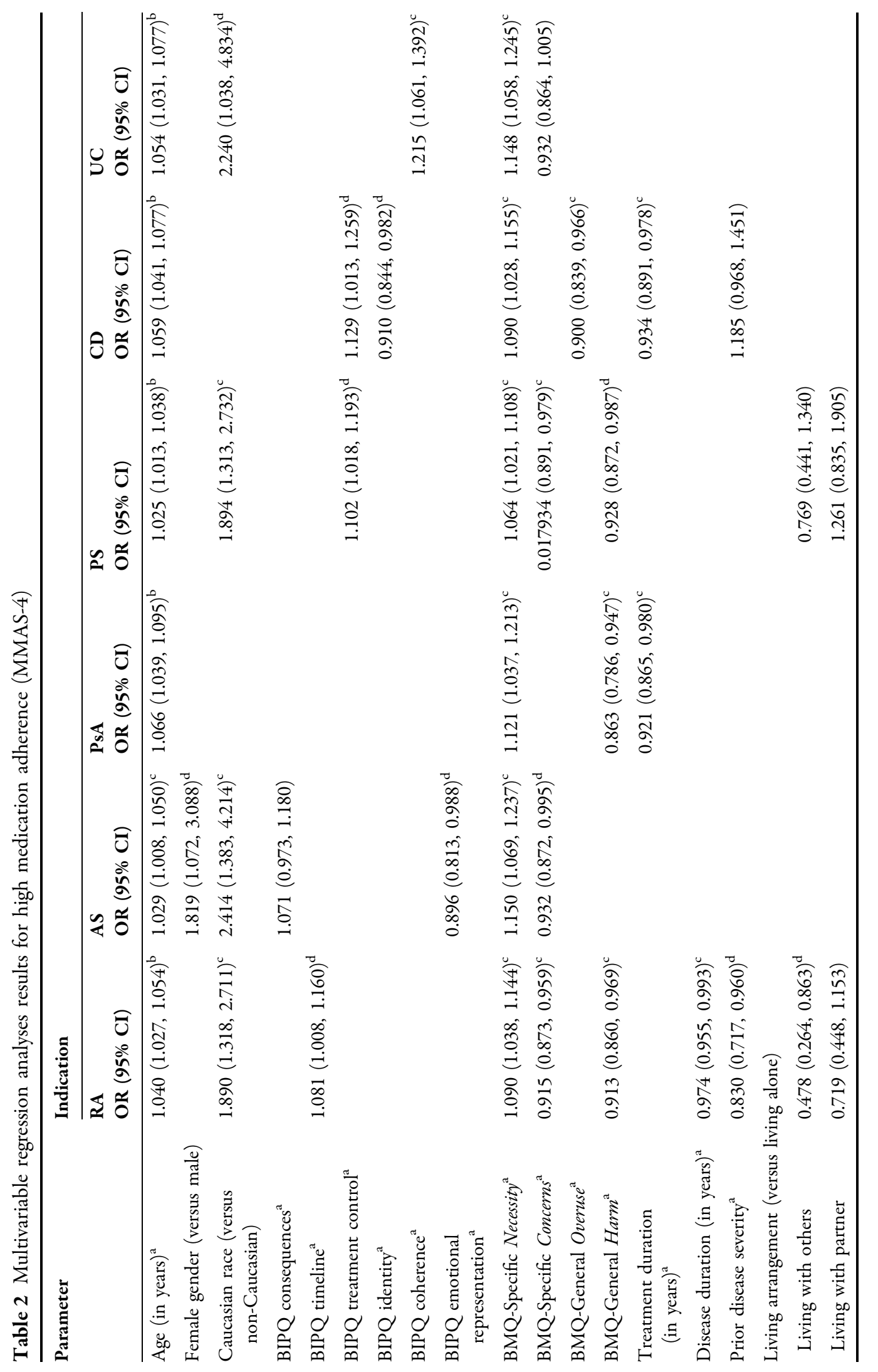




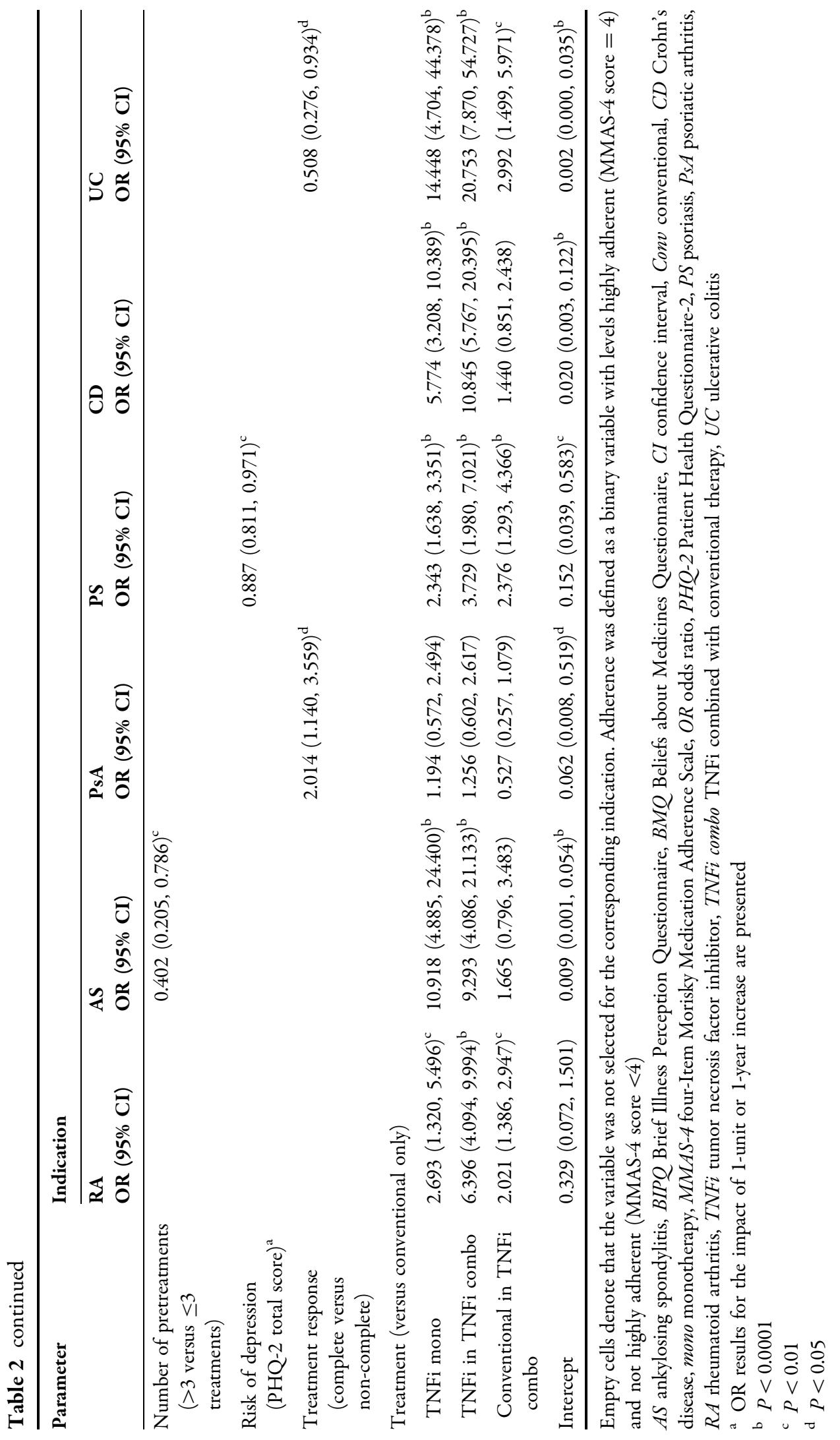




\section{Multivariable Regression Analyses}

\section{Logistic Multivariable Regression Analyses: MMAS-4 Adherence Scores}

In all IMIDs except PsA, TNFi monotherapy or combination therapy was associated with 2- to 21-fold higher likelihood of high medication adherence compared with conventional only therapy (Table 2; Fig. S2). A direct positive association of older age and greater BMQ-Specific Necessity score with high medication adherence was observed in all six IMIDs, while Caucasian race was associated with greater medication adherence in four of six IMIDs (Table 2; Fig. S2). Higher

\section{Factors associated with higher BMQ-}

Specific Necessity score:

- Age $\uparrow^{\star+1}$

- BIPQ Timeline score $\uparrow$ ("How long do you think your illness will continue?') "*t

- BIPQ Treatment control score $\uparrow$ ("How much do you think your treatment can help your illness?") **

- BIPQ Illness concern score $\uparrow$ ("How concerned are you about your illness?') $\$ 2$

- Risk of depression (PHQ-2 total score) $\uparrow^{\text {t+4 }}$

- Complete treatment response $\uparrow \star 3$

- TNFi monotherapy *4

- TNFi therapy within TNFi combo **\#

Fig. 3 Summary of factors associated with higher treatment necessity beliefs and/or medication adherence in at least four of six IMIDs (results from multivariable regression analyses). High adherence: MMAS- $4=4$; $\uparrow=$ higher/older; three filled diamonds positive association identified in all of the six IMIDs; two filled diamonds positive association identified in four to five of the six IMIDs, but variable excluded from testing in at least one indication; one filled diamond positive association identified in four to five of the six IMIDs, but non-significant result in at least one indication; ${ }^{1}$ variable excluded from testing in PsA; ${ }^{2}$ variable excluded from testing in $\mathrm{CD}$ and $\mathrm{UC} ;{ }^{3}$ variable excluded from testing in
BMQ-Specific Concerns scores were associated with less medication adherence in RA, AS, and PS. Factors associated with higher treatment necessity beliefs and/or higher medication adherence in at least four IMIDs are summarized in Fig. 3.

\section{Factors Affecting Necessity Beliefs and Concerns Towards Current IMID}

\section{Medication}

Higher BIPQ timeline scores (all IMIDs), BIPQ treatment control scores (all IMIDs), and BIPQ illness concerns (RA, AS, PsA, and PS) were associated with greater BMQ-Specific Necessity scores (Table S7). Higher scores on the BIPQ

\section{Factors associated with high MMAS-4} adherence:

- Age $\uparrow\left(\mathrm{OR}=1.03-1.07^{\S}\right)$ **

- Caucasian ethnicity $(\mathrm{OR}=1.89-2.41) \leftrightarrow 5$

- Treatment necessity beliefs $\uparrow^{\diamond}$ $\left(\mathrm{OR}=1.06-1.15^{\S}\right)$

- TNFi monotherapy $(\mathrm{OR}=2.34-14.45) \bullet 6$

- TNFi therapy within TNFi combo $(\mathrm{OR}=3.73-20.75){ }^{6}$

PsA, result non-significant for $\mathrm{CD} ;{ }^{4}$ result non-significant for RA and PsA; ${ }^{5}$ variable excluded from testing in PsA and $\mathrm{CD} ;{ }^{6}$ non-significant result in PsA; ${ }^{\#}$ versus conventional only therapy; ${ }^{5} \mathrm{OR}$ results for the impact of a 1-year or 1-unit increase presented; empty diamond as determined by BMQ-Specific Necessity. BIPQ Brief Illness Perception Questionnaire, $B M Q$ Beliefs about Medicines Questionnaire, $C D$ Crohn's disease, IMID immune-mediated inflammatory disease, $M M A S-4$ four-item Morisky Medication Adherence Scale, OR odds ratio, PHQ-2 Patient Health Questionnaire-2, $P_{s} A$ psoriatic arthritis, $R A$ rheumatoid arthritis, $T N F i$ tumor necrosis factor inhibitor, $U C$ ulcerative colitis 
illness concern and emotional impact of illness scales (all IMIDs) were associated with higher BMQ-Specific Concerns scores, while lower BIPQ coherence score (all IMIDs) and lower BIPQ treatment control scores (all IMIDs except AS) were associated with lower treatment concerns (Table S8). "Complete treatment response" and treatment type were the only disease- or treatment-related factors with a significant impact on treatment necessity beliefs or treatment concerns in four or more IMIDs. TNFi in combination therapy (all IMIDs) and TNFi monotherapy (AS, PS, CD, and UC) were associated with greater BMQ-Specific Necessity scores. Increased depressive symptoms were associated with higher BMQ-Specific Concerns and Necessity scores, and Caucasian race was associated with lower treatment concerns.

\section{DISCUSSION}

Various "patient-supporting" approaches (e.g., telephone follow-up and supportive care) have been established and tested in order to improve medication adherence. Many studies on the impact of patient support methods have been small in size and produced mixed results $[35,36]$. To devise successful programs and to provide targeted adherence support, it is crucial to understand key drivers for enhanced treatment adherence in patients with IMIDs. To our knowledge, ALIGN is the first study to analyze the association between patients' beliefs about their illness, as well as about their systemic medications, and self-reported adherence patterns in a large multi-country population with more than 7000 patients across six different IMIDs. Four validated questionnaires were used in the ALIGN study, allowing examination of the effects of various factors on patients' medication-related beliefs and treatment adherence in a diverse set of multivariable regression analyses. Identifying the factors that have the most effect on adherence to therapy can help develop models to predict the risk of non-adherence for patients with specific IMIDs. Targeted intervention can then be designed to improve adherence to therapy in patients at high risk of non-adherence.

Several key findings from the ALIGN study provide new insight into beliefs and concerns of patients with IMIDs towards their systemic medication. BMQ-Specific Concerns scores for different treatment groups across indications were in a similar range, suggesting that patients today are not significantly more concerned about potential side effects of TNFi than they are about those associated with conventional systemic treatments. However, the results for BMQ-General revealed that a significant proportion of patients still had negative beliefs about medication in general (e.g., perception of all medicines to be poison and of most medicines to be addictive).

Multivariable regression analyses revealed that, in all IMIDs except PsA, TNFi monotherapy or in combination therapy was associated with a 2- to 21-fold higher likelihood of high medication adherence compared with conventional only therapy. "Complete response to current treatment" was identified as an independent predictor of either high or low adherence in patients with PsA or UC, respectively. However, only few clinical parameters were associated with higher treatment necessity beliefs or higher medication adherence, possibly because the majority of patients displayed low disease activity and had responded to their treatment regimen. These results generally correspond with published data from Dutch investigations for RA, in which clinical parameters did not significantly influence non-adherence $[19,20]$. 
In ALIGN, perceptions about the necessity of the medication rather than concern beliefs affected high medication adherence in a majority of the IMIDs. These observations are consistent with results from four [19, 20, 37, 38] of the six [19, 20, 28, 37-39] previous studies assessing associations between beliefs about medication and non-adherence in patients with RA. Our data suggest that necessity beliefs about medication are a prerequisite for taking medicines, and that necessity beliefs seem to outweigh concerns about the medication. A systemic review and meta-analysis across various diseases supports this observation [25], although, in our regression analysis, a relationship between higher concerns and less adherence was noted only in RA, AS, and PS.

Across all six IMIDs, patients with higher medication necessity beliefs were those who believed more strongly that their condition would be longer lasting and was more controllable by treatment, and who had greater concerns about their illness. Direct associations of the perception of the illness to continue longer/forever (in RA) and the perception of the treatment to help (in PS and CD) with higher medication adherence, and of experiencing more symptoms with lower adherence, were seen with certain IMIDs but not all, indicating a rather indirect association of several BIPQ items with treatment adherence via their effect on treatment necessity beliefs in the majority of IMIDs. In addition, in at least four of the six IMIDs, older age and Caucasian race were associated with better medication adherence. Age or race/ethnicity as factors affecting medication adherence were noted in patients with RA or other immune diseases in previous studies [18, 39-44]. In our study, Caucasians in general harbored significantly less concerns and overuse beliefs but not higher treatment necessity beliefs compared with non-Caucasians.
One limitation of the ALIGN study was that illness perception, beliefs about medication, risk of depression, and adherence results were based on self-reported outcomes that may be influenced by self-presentational and recall biases. Patients may overestimate the extent of their adherence in an attempt to "please the doctor," or if they believe that admitting to non-adherence may result in adverse judgments. However, in our study patients' responses were confidential; thus, the risk of overestimating adherence to "please the doctor" was reduced. Another caveat is that the patients' responses to the specific questions in the questionnaires used in this study may not capture all the complexities of patients' feelings. Additionally, for feasibility reasons, no validated clinical scores were used to assess disease severity or response to therapy, and no uniform definitions or confirmations were used for diagnosing the IMIDs. Furthermore, confounding factors (e.g., disease severity, treatment response, and number of co-morbidities) may not be equally distributed between the groups being compared in this non-randomized study, which could lead to bias and subsequent misinterpretation. However, in our multivariable regression analyses, we controlled for various confounding factors so that such effects would be minimized and false interpretation could be avoided. Although different adherence measures were applied in other studies, limiting comparability with results from ALIGN, the percentages of patients with PS or CD treated with TNFi monotherapy and combination therapy who reported high adherence based on the MMAS- 4 corresponded to the adherence levels for biologics reported in the same IMIDs by other authors [8, 45]. Another limitation is the cross-sectional rather than prospective design of this study. However, the data obtained allowed the formulation of a new hypothesis which can 
be tested prospectively in future studies. Additionally, the proportion of patients receiving TNFi therapy versus conventional therapy in this study may not be representative of typical practice. Finally, information about the percentage of screened patients that agreed to participate in this study was not collected. Despite its limitations, the large, global, multicenter ALIGN study provides valuable insight into factors affecting treatment-related beliefs and medication adherence in patients with IMIDs.

\section{CONCLUSION}

ALIGN is the first large cross-sectional study to provide extensive data on the impact of various factors, including patients' beliefs about medication, on medication adherence in patients diagnosed with one of the six different IMIDs. Treatment necessity beliefs are higher than concerns about current medication in IMID patients with well-controlled disease. Illness perceptions appear to have a greater impact on necessity beliefs about medication than clinical parameters. Older age, greater treatment necessity beliefs, and TNFi therapy are associated with high self-reported medication adherence across all IMIDs. Insights from the ALIGN study may help develop tools to screen for IMID patients at risk of poor adherence.

\section{ACKNOWLEDGEMENTS}

Sponsorship, article processing charges, and the open access charge for this study were funded by AbbVie Inc. The authors would like to thank Donald Morisky, ScD, ScM, MSPH, UCLA Fielding School of Public Health, Department of Community Health Sciences, Los Angeles, California, USA, for permission to use the four-item Morisky Medication Adherence Scale (MMAS-4). Use of the CMMAS is protected by US copyright laws. Permission for use is required. A license agreement is available from Professor Donald E. Morisky, Department of Community Health Sciences, UCLA Fielding School of Public Health, 650 Charles E. Young Drive South, Los Angeles, CA 90095-1772, dmorisky@ucla.edu. The authors also thank Stefanie Kalus, PhD, GKM, Munich, Germany, for her statistical support, and Rita Tarzynski-Potempa, MD, AbbVie, for her scientific review. Furthermore, the authors would like to thank all investigators for their contribution to the success of the ALIGN study. Editorial assistance in the preparation of this manuscript was provided by Patrick Little, PhD, and Michael J. Theisen, PhD, of Complete Publication Solutions, LLC, North Wales, Pennsylvania, USA. Support for this assistance was funded by AbbVie Inc. AbbVie contributed to the study design, and was involved in the collection, analysis, and interpretation of the data, and in the writing, review, and approval of the publication. All named authors meet the International Committee of Medical Journal Editors (ICMJE) criteria for authorship for this manuscript, take responsibility for the integrity of the work as a whole, and have given final approval for the version to be published.

Disclosures. Pierre Michetti has received consulting fees from MSD, AbbVie, Calypso, Merck-Serono, Ferring, Vifor, UCB Pharma, Takeda, Nestlé Health Sciences, and Pfizer, lecture fees from MSD, AbbVie, Falk, AstraZeneca, Takeda, Ferring, Vifor, UCB Pharma, grants from MSD, UCB Pharma, and Takeda, and personal fees from AbbVie during the conduct of the study; John Weinman is a part-time employee of Atlantis Healthcare, who develop patient support programs for many 
pharmaceutical companies, and has received consulting and lecture fees from AbbVie, Boehringer Ingelheim, Ferring, Roche, and Sanofi; Ulrich Mrowietz has received speaker and advisory board honoraria from AbbVie, grants from AbbVie for educational projects, and other compensation from AbbVie for clinical trial research; Josef Smolen has received grants and personal fees from AbbVie, Lilly, MSD, Pfizer, and Roche and personal fees from Amgen, AstraZeneca, Astro, Celgene, GlaxoSmithKline, Janssen, MedImmune, Novartis, Samsung, and UCB; Laurent Peyrin-Biroulet has received consulting fees from Merck, AbbVie, Janssen, Genentech, Mitsubishi, Ferring, Norgine, Tillotts, Vifor, Therakos, Pharmacosmos, Pilège, BMS, UCB Pharma, Hospira, Celltrion, Takeda, Biogaran, Boehringer Ingelheim, Lilly, Pfizer, H.A.C. Pharma, and Index Pharmaceuticals, and lecture fees from Merck, AbbVie, Takeda, Janssen, Ferring, Norgine, Tillotts, Vifor, Therakos, Mitsubishi, and H.A.C. Pharma; Edouard Louis has received grants from AbbVie and MSD, personal fees from AbbVie, MSD, Ferring, Takeda, Celltrion, Mundipharma, Hospira, and Janssen, and non-financial support from AbbVie and MSD; Dieter Schremmer is an employee of the contract research organization GKM Gesellschaft für Therapieforschung $\mathrm{mbH}$; Namita Tundia is an AbbVie employee and shareholder of AbbVie stock; Pascal Nurwakagari was an AbbVie employee and shareholder of AbbVie stock at the time of the study; Nicole Selenko-Gebauer is an AbbVie employee and shareholder of AbbVie stock.

Compliance with Ethics Guidelines. All procedures followed were in accordance with the ethical standards of the responsible committee on human experimentation (institutional and national) and with the Helsinki Declaration of 1964, as revised in
2013. Informed consent was obtained from all patients for being included in the study. This study was approved by the local ethics committee of each participating country.

Data Availability. The datasets during and/or analyzed during the current study are available from the corresponding author on reasonable request.

Open Access. This article is distributed under the terms of the Creative Commons Attribution-NonCommercial 4.0 International License (http://creativecommons.org/licenses/ by-nc/4.0/), which permits any noncommercial use, distribution, and reproduction in any medium, provided you give appropriate credit to the original author(s) and the source, provide a link to the Creative Commons license, and indicate if changes were made.

\section{REFERENCES}

1. Bluett J, Morgan C, Thurston L, et al. Impact of inadequate adherence on response to subcutaneously administered anti-tumour necrosis factor drugs: results from the Biologics in Rheumatoid Arthritis Genetics and Genomics Study Syndicate cohort. Rheumatology (Oxford). 2015;54:494-9.

2. Waimann CA, Marengo MF, de Achaval S, et al. Electronic monitoring of oral therapies in ethnically diverse and economically disadvantaged patients with rheumatoid arthritis: consequences of low adherence. Arthritis Rheum. 2013;65:1421-9.

3. Contreras-Yanez I, Ponce De Leon S, Cabiedes J, Rull-Gabayet M, Pascual-Ramos V. Inadequate therapy behavior is associated to disease flares in patients with rheumatoid arthritis who have achieved remission with disease-modifying antirheumatic drugs. Am J Med Sci. 2010;340:282-90.

4. Kane SV, Robinson A. Review article: understanding adherence to medication in ulcerative colitisinnovative thinking and evolving concepts. Aliment Pharmacol Ther. 2010;32:1051-8. 
5. Kane S, Huo D, Aikens J, Hanauer S. Medication nonadherence and the outcomes of patients with quiescent ulcerative colitis. Am J Med. 2003;114:39-43.

6. Feagins LA, Iqbal R, Spechler SJ. Case-control study of factors that trigger inflammatory bowel disease flares. World J Gastroenterol. 2014;20:4329-34.

7. Zaghloul SS, Goodfield MJ. Objective assessment of compliance with psoriasis treatment. Arch Dermatol. 2004;140:408-14.

8. Kane SV, Chao J, Mulani PM. Adherence to infliximab maintenance therapy and health care utilization and costs by Crohn's disease patients. Adv Ther. 2009;26:936-46.

9. Feagan BG, Kozma CM, Slaton TL, Olson WH, Wan GJ. Healthcare costs for Crohn's disease patients treated with infliximab: a propensity weighted comparison of the effects of treatment adherence. J Med Econ. 2014;17:872-80.

10. Mitra D, Hodgkins P, Yen L, Davis KL, Cohen RD. Association between oral 5-ASA adherence and health care utilization and costs among patients with active ulcerative colitis. BMC Gastroenterol. 2012;12:132.

11. De Vera MA, Mailman J, Galo JS. Economics of non-adherence to biologic therapies in rheumatoid arthritis. Curr Rheumatol Rep. 2014;16:460.

12. Tang B, Rahman M, Waters HC, Callegari P. Treatment persistence with adalimumab, etanercept, or infliximab in combination with methotrexate and the effects on health care costs in patients with rheumatoid arthritis. Clin Ther. 2008;30:1375-84.

13. World Health Organization. Adherence to long-term therapies: evidence for action. Geneva: World Health Organization; 2003.

14. Thorneloe RJ, Bundy C, Griffiths CE, Ashcroft DM, Cordingley L. Adherence to medication in patients with psoriasis: a systematic literature review. Br J Dermatol. 2013;168:20-31.

15. Fidder $H H$, Singendonk $M M$, van der Have $M$, Oldenburg B, van Oijen MG. Low rates of adherence for tumor necrosis factor-alpha inhibitors in Crohn's disease and rheumatoid arthritis: results of a systematic review. World J Gastroenterol. 2013;19:4344-50.

16. Harrold LR, Andrade SE. Medication adherence of patients with selected rheumatic conditions: a systematic review of the literature. Semin Arthritis Rheum. 2009;38:396-402.
17. Pascual-Ramos V, Contreras-Yanez I. Motivations for inadequate persistence with disease modifying anti-rheumatic drugs in early rheumatoid arthritis: the patient's perspective. BMC Musculoskelet Disord. 2013;14:336.

18. Viller F, Guillemin F, Briancon S, Moum T, Suurmeijer T, van den Heuvel W. Compliance to drug treatment of patients with rheumatoid arthritis: a 3 year longitudinal study. J Rheumatol. 1999;26:2114-22.

19. Zwikker HE, van Dulmen S, den Broeder AA, van den Bemt BJ, van den Ende $\mathrm{CH}$. Perceived need to take medication is associated with medication non-adherence in patients with rheumatoid arthritis. Patient Prefer Adherence. 2014;8:1635-45.

20. van den Bemt BJ, van den Hoogen FH, Benraad B, Hekster YA, van Riel PL, van Lankveld W. Adherence rates and associations with nonadherence in patients with rheumatoid arthritis using disease modifying antirheumatic drugs. J Rheumatol. 2009;36:2164-70.

21. Pasma A, van't Spijker A, Hazes JM, Busschbach JJ, Luime JJ. Factors associated with adherence to pharmaceutical treatment for rheumatoid arthritis patients: a systematic review. Semin Arthritis Rheum. 2013;43:18-28.

22. Feagan BG, MacDonald JK. Oral 5-aminosalicylic acid for maintenance of remission in ulcerative colitis. Cochrane Database Syst Rev. 2012;10:CD000544.

23. Lopez A, Billioud V, Peyrin-Biroulet C, Peyrin-Biroulet L. Adherence to anti-TNF therapy in inflammatory bowel diseases: a systematic review. Inflamm Bowel Dis. 2013;19:1528-33.

24. McHorney CA, Spain CV. Frequency of and reasons for medication non-fulfillment and non-persistence among American adults with chronic disease in 2008. Health Expect. 2011;14:307-20.

25. Horne R, Chapman SC, Parham R, Freemantle N, Forbes A, Cooper V. Understanding patients' adherence-related beliefs about medicines prescribed for long-term conditions: a meta-analytic review of the Necessity-Concerns Framework. PLoS One. 2013;8:e80633.

26. Horne R, Parham R, Driscoll R, Robinson A. Patients' attitudes to medicines and adherence to maintenance treatment in inflammatory bowel disease. Inflamm Bowel Dis. 2009;15:837-44.

27. Horne R, Weinman J. Patients' beliefs about prescribed medicines and their role in adherence 
to treatment in chronic physical illness. J Psychosom Res. 1999;47:555-67.

28. Neame R, Hammond A. Beliefs about medications: a questionnaire survey of people with rheumatoid arthritis. Rheumatology (Oxford). 2005;44:762-7.

29. Horne R, Weinman J, Hankins M. The Beliefs about Medicines Questionnaire: the development and evaluation of a new method for assessing the cognitive representation of medication. Psychol Health. 1999;14:1-24.

30. Morisky DE, DiMatteo MR. Improving the measurement of self-reported medication nonadherence: response to authors. J Clin Epidemiol. 2011;64:255-7 (discussion 8-63).

31. Morisky DE. Donald Morisky's website: MMAS scale. http://dmorisky.bol.ucla.edu/MMAS_scale. html. Accessed 19 Feb 2016.

32. Broadbent E, Petrie KJ, Main J, Weinman J. The Brief Illness Perception Questionnaire. J Psychosom Res. 2006;60:631-7.

33. Kroenke K, Spitzer RL, Williams JB. The Patient Health Questionnaire-2: validity of a two-item depression screener. Med Care. 2003;41:1284-92.

34. Morisky DE, Green LW, Levine DM. Concurrent and predictive validity of a self-reported measure of medication adherence. Med Care. 1986;24:67-74.

35. Nieuwlaat R, Wilczynski N, Navarro $\mathrm{T}$, et al. Interventions for enhancing medication adherence. Cochrane Database Syst Rev. 2014;11:CD000011.

36. Galo JS, Mehat P, Rai SK, Avina-Zubieta A, De Vera MA. What are the effects of medication adherence interventions in rheumatic diseases: a systematic review. Ann Rheum Dis. 2016;75:667-73.

37. Treharne GJ, Lyons AC, Kitas GD. Medication adherence in rheumatoid arthritis: effects of psychosocial factors. Psychol Health Med. 2004;9:337-49.

38. de Thurah A, Norgaard $M$, Johansen MB, Stengaard-Pedersen K. Methotrexate compliance among patients with rheumatoid arthritis: the influence of disease activity, disease duration, and co-morbidity in a 10-year longitudinal study. Scand J Rheumatol. 2010;39:197-205.

39. Morgan C, McBeth J, Cordingley L, et al. The influence of behavioural and psychological factors on medication adherence over time in rheumatoid arthritis patients: a study in the biologics era. Rheumatology (Oxford). 2015;54:1780-91.

40. Bliddal $H$, Eriksen SA, Christensen R, et al. Adherence to methotrexate in rheumatoid arthritis: a Danish nationwide cohort study. Arthritis. 2015;2015:915142.

41. Quinlan P, Price KO, Magid SK, Lyman S, Mandl LA, Stone PW. The relationship among health literacy, health knowledge, and adherence to treatment in patients with rheumatoid arthritis. HSS J. 2013;9:42-9.

42. Salt E, Frazier SK. Predictors of medication adherence in patients with rheumatoid arthritis. Drug Dev Res. 2011;72:756-63.

43. Solomon DH, Tonner C, Lu B, et al. Predictors of stopping and starting disease-modifying antirheumatic drugs for rheumatoid arthritis. Arthritis Care Res (Hoboken). 2014;66:1152-8.

44. van Mierlo T, Fournier R, Ingham M. Targeting medication non-adherence behavior in selected autoimmune diseases: a systematic approach to digital health program development. PLoS One. 2015;10:e0129364.

45. Kamangar F, Isip L, Bhutani T, et al. How psoriasis patients perceive, obtain, and use biologic agents: survey from an academic medical center. J Dermatolog Treat. 2013;24:13-24. 